\title{
The Neutronic Check Point: fast neutron transmission measurements to detect explosives in vehicles.
}

\section{S. Pesente, G. Nebbia, D. Fabris, M. Lunardon, S. Moretto}

INFN and Dipartimento di Fisica dell' Università di Padova

Via Marzolo 8, I-35131 Padova, Italy

\section{G. Viesti*}

INFN and Dipartimento di Fisica dell' Università di Padova

Via Marzolo 8, I-35131 Padova, Italy

E-mail: giuseppe.viesti@pd.infn.it

\begin{abstract}
The detection of Improvised Explosive Devices hidden in vehicles is one major task in the prevention of terrorist actions. Detailed GEANT Monte Carlo simulations have been recently performed for transmitted and scattered neutrons in the case of a car having explosive hidden in the trunk. Simulation results are discussed. Considerations about the use of the neutron inspection in connection with other techniques and licensing problems are also presented.
\end{abstract}

International Workshop on Fast Neutron Detectors

University of Cape Town, South Africa

April 3 - 6, 2006

* Speaker 


\section{Introduction}

The threat of terrorist use of explosive devices and chemical, biological or radioactive agents has become realistic since the bombing of Pan Am Flight 103 at Lockerbie, Scotland on December 21, 1988 and the SARIN attack in the Tokyo subway system on March 20, 1995. A long series of events started more recently with the tragic events of September 11, 2001, the latest being the recent bombings in Madrid and London. The possibility of further terrorist actions against civil populations is one of the most important issues on the international political agenda [1]. Security Research will be one priority in the next Seventh Framework Program of the European Union. In the meantime, a Preparatory Action on Security Research has been in progress since 2004 [2].

The prevention of the terrorist actions involves several different tasks. A specific aspect of the problem is the detection of hidden explosive in vehicles that are used in suicide bomber attacks worldwide. The fast scan of vehicles operated at sufficiently large distance from the target itself, to avoid damage in case of explosion, is a mandatory requirement.

$\mathrm{X}$-ray or gamma-ray based systems provide good precision density measurements with high resolution three-dimensional images, but only gross information about the elemental content of the inspected item (low $\mathrm{Z}$ vs. high $\mathrm{Z}$ discrimination) with limits due to the penetration depth of the radiation [3]. Neutron interrogation offers the possibility of measuring the elemental density of most elements in materials independent from their particular structure and the use of neutron induced reactions for nondestructive bulk elemental analysis is well documented [4,5]. All neutrons, in particular fast neutrons, are well suited to explore large volume samples because of their high penetration in bulk material. The detection of hidden explosives can be achieved by determining the elemental ratio of $\mathrm{C}, \mathrm{N}, \mathrm{O}$ nuclei from the intensity of the characteristic gamma-rays produced in fast neutron induced reactions. This technique, known as Fast Neutron Analysis (FNA), requires measuring times of several minutes and large equipments so that it seems not suited to be applied in the vehicle inspection context. Less specific but rapid signature of the presence of large quantity of hydrogenated materials can be obtained by looking at the transmission and scattering of fast neutrons and adding the neutron "image" to the one obtained form X-rays, Such technique has been recently proposed for scanning air cargo containers by CSIRO in Australia [6].

In this paper results obtained from Monte Carlo simulations of vehicle inspections will be presented and discussed with the objective of developing a practical conceptual design of a vehicle check point.

\section{Monte Carlo simulations}

The quantity of explosive material used in attacks with Improvised Explosives Devices hidden in vehicles ranges from few kilograms to several tons, depending of the type of target. A large quantity of hydrogen rich material can be detected in very short time by looking at the transmitted and scattered neutrons. This technique does not provide precise information about the chemical composition of the voxel, but could be used to make a fast first level scanning of vehicles discriminating the type of material that are causing the neutron absorption.

Detailed GEANT Monte Carlo simulations [7] have been recently performed in the case of a car having explosive hidden in the trunk. We have simulated the use of a Tagged Neutron Inspection System (TNIS) using the D+T neutron source reaction [8], with a 64 pixel matrix embedded into the system to detect the associated alpha particles and tag the neutron beam. 
In the simulation, the car is approximated by a stainless steel and plastic box having dimensions $4.5 \times 1.8 \times 1.5 \mathrm{~m}^{3}$. In the car we have simulated the presence of a plastic baggage volume, a tank filled with fuel, the tires, the front and back seats and the engine. The distance between the neutron producing target and the center of the car is $120 \mathrm{~cm}$.

Tagged neutrons transmitted trough the vehicle are detected in coincidence with the associated alpha particles by using an array of position sensitive neutron detectors $\left(\mathrm{N}_{\mathrm{T}}\right)$ covering an area of $110 \times 110 \mathrm{~cm}^{2}$. Four large pad detectors $\left(\mathrm{N}_{\mathrm{D} 1}, \mathrm{~N}_{\mathrm{D} 2}, \mathrm{~N}_{\mathrm{D} 3}, \mathrm{~N}_{\mathrm{D} 4}\right)$, having an area of $120 \mathrm{x}$ $50 \mathrm{~cm}^{2}$ each are used to detect scattered neutrons at the road level below the car.

The car and the inspection systems as folded in the Monte Carlo simulations are shown in Figure 1. Each point in the car scan corresponds to the emission of $8 \times 10^{6}$ neutrons over $4 \pi$. To reproduce the scanning of the car, the neutron detectors and the neutron generator were moved along the $\mathrm{x}$ axis in steps of $40 \mathrm{~cm}$.

With the neutron detector placed behind the car we can measure the attenuation of the neutrons passing through different materials. We define the neutron Attenuation Factor (ATF) as the ratio $\left(A_{\max }-A_{i}\right) / A_{\max }$, where $A_{\max }$ is the maximum number of "unperturbed" neutrons (i.e. the ones in the time-of-flight window corresponding to $14 \mathrm{MeV}$ ) that are hitting the neutron detector during the scanning of the car and $A_{i}$ is the number of unperturbed neutrons hitting the neutron detector, when the generator is placed in the position i along the vehicle.

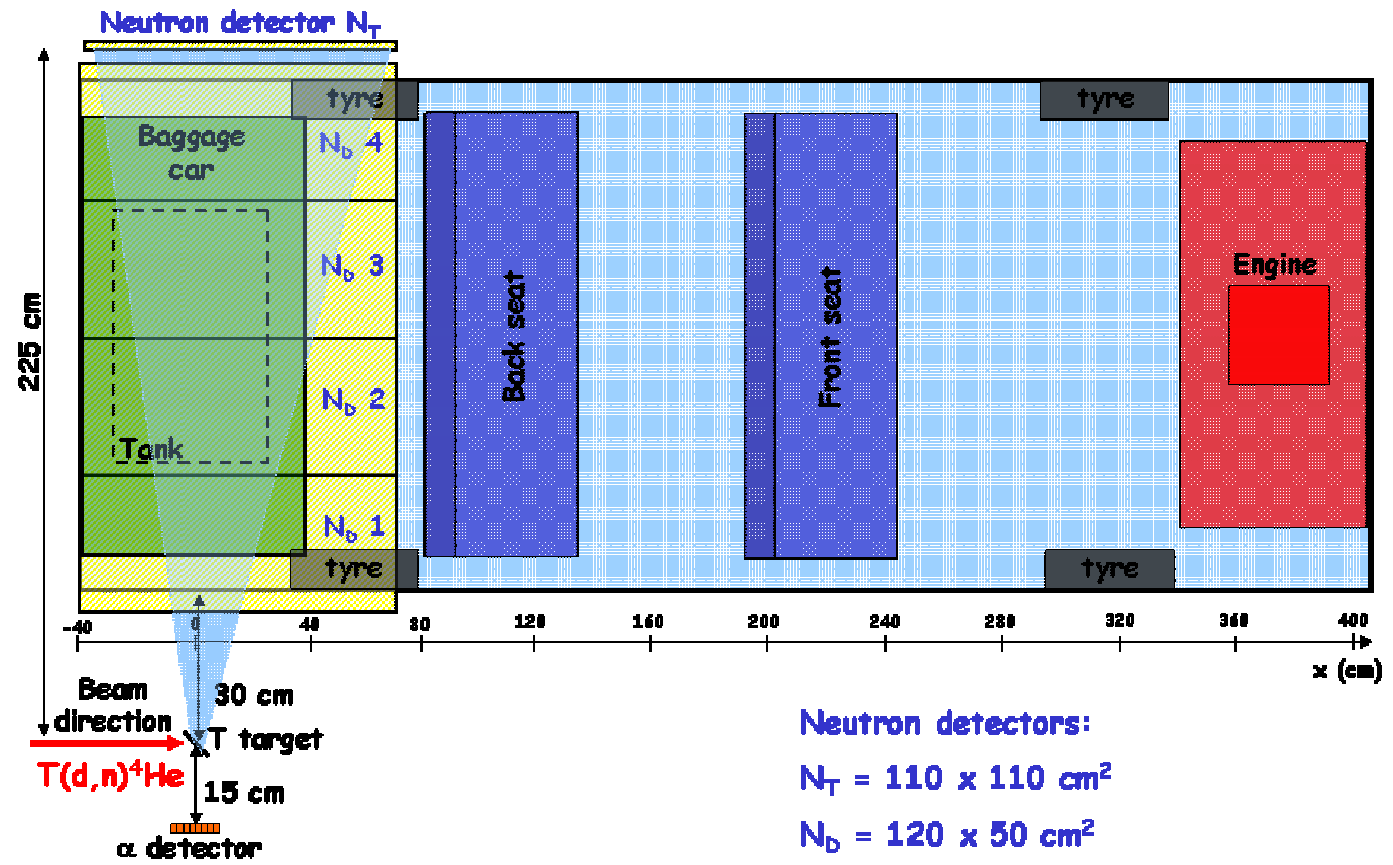

Figure 1 Lay-out of the simulated system with the neutron generator, the car and two neutron detection array

With the neutron detectors placed under the car $\left(\mathrm{N}_{\mathrm{D}}\right)$ it is possible to estimate the number of neutrons scattered backward (i.e. in the detectors $N_{D 1}$ and $N_{D 2}$ ) and forward (i.e. in the detectors $\mathrm{N}_{\mathrm{D} 3}$ and $\mathrm{N}_{\mathrm{D} 4}$ ) with respect to the neutron beams. We have defined as Asymmetry Factor (ASF) the ratio $\left(A_{D 1}+A_{D 2}\right) /\left(A_{D 3}+A_{D 4}\right)$, where $A_{D i}$ are the counts in each $N_{D i}$ neutron detector pad.

Both the observables, ATF and ASF, are used to discriminate the presence of light $\mathrm{Z}$ material, as in the case of explosive, from other thick absorbers, as the vehicle engine. 

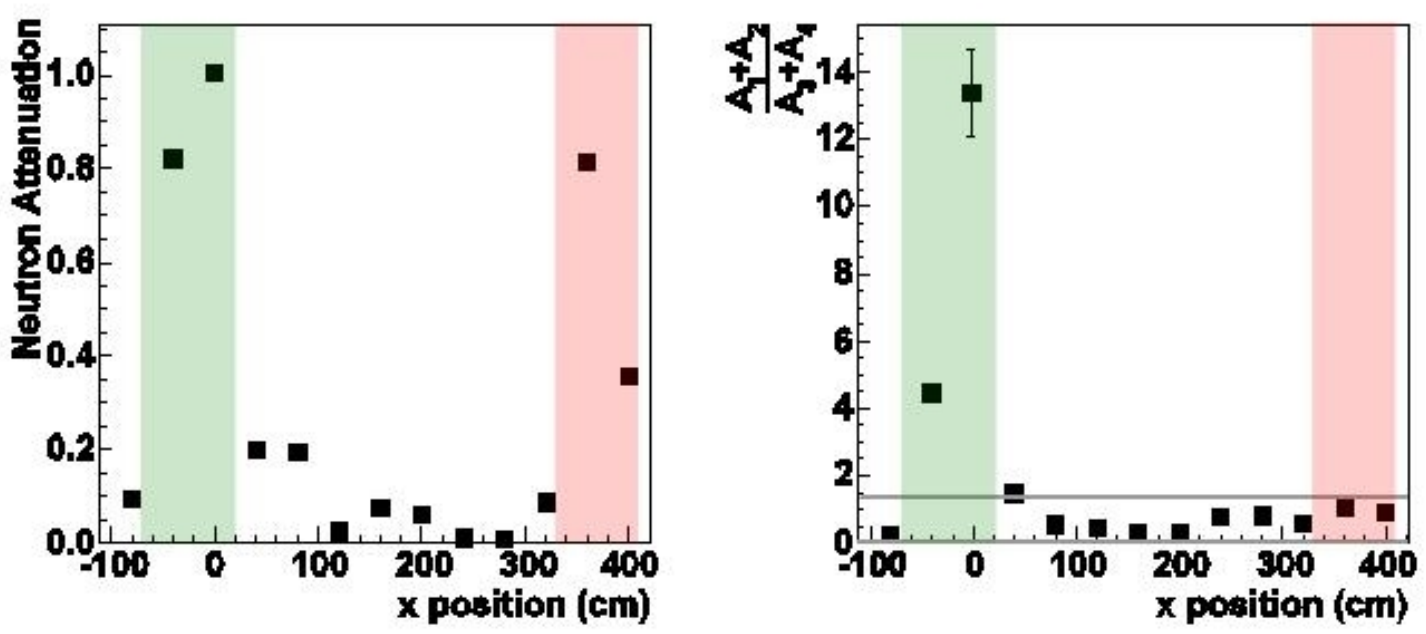

Figure 2 Left panel: Neutron Attenuation Factor as a function of the neutron generator position. Right panel: Asymmetry Factor as a function of the neutron generator position. The two dashed area correspond to the positions of the hidden explosive (light blue) and the car engine (pink). For details see the text.
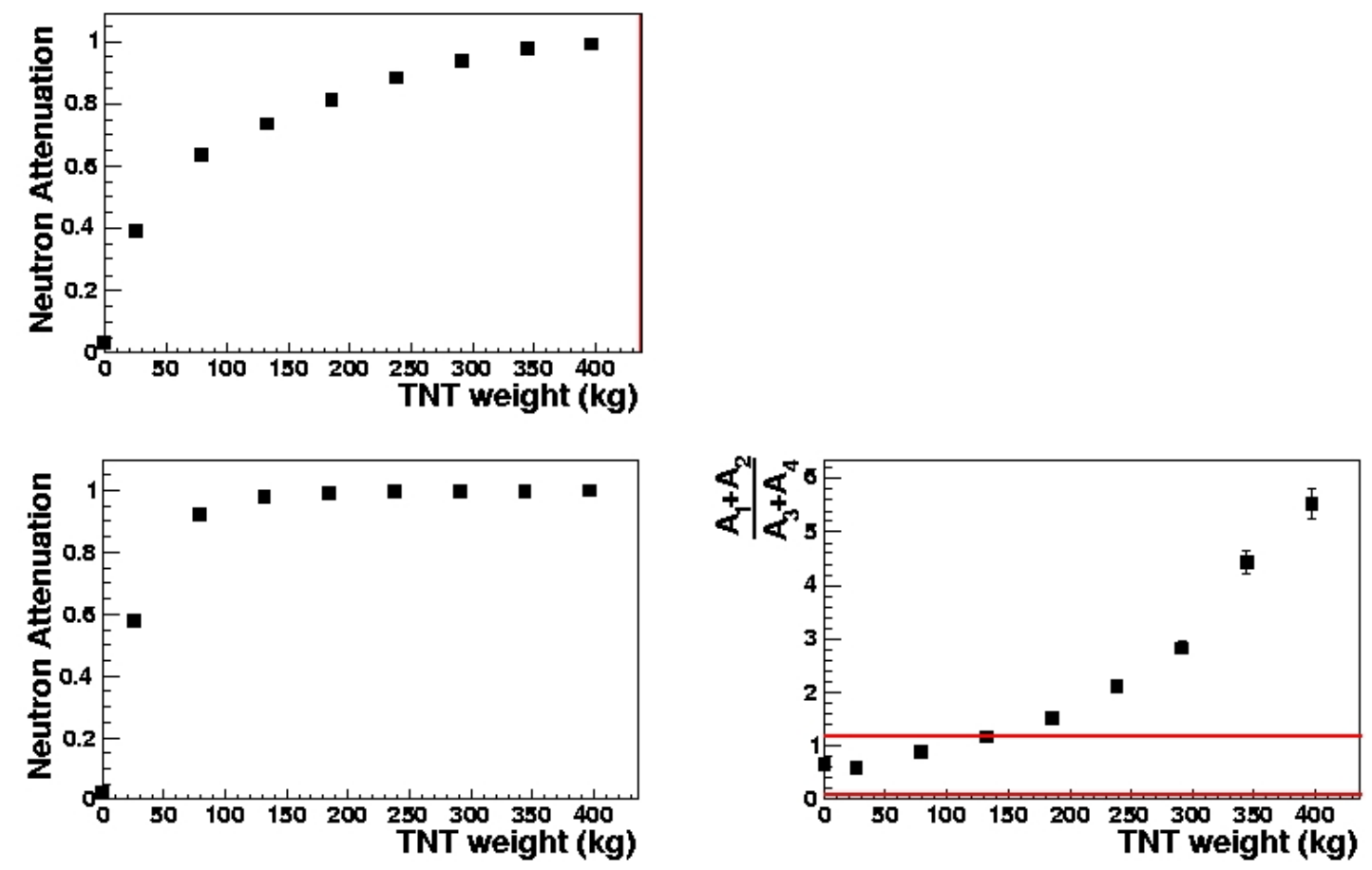

Figure 3 Neutron Attenuation Factor and Asymmetry Factor as a function of the weight of the explosive hidden in the trunk. Calculations correspond to the position $\mathrm{x}=0$ along the car (see Fig.1). Upper panel wide beam. Lower panel narrow beam. For details see the text. 
In the left panel of Figure 2 we have reported the value of the neutron Attenuation Factor as a function of the scanning position along the car when the trunk is completely full of explosive. Two regions with a strong attenuation are identified, which correspond to the scanning of the trunk and of the engine. The values of the Asymmetry Factor as a function of the neutron generator position are shown in the right panel of Figure 2. The Asymmetry Factor varies generally in the range from 0 . to 1.2 for all positions with the exception of the trunk filled with TNT where it is much larger. It means that the ASF value can be used to discriminate the presence of low $\mathrm{Z}$ material inside the vehicle.

A number of simulations have been also performed by changing the dimensions of the TNT sample placed at the centre of the trunk. The sample was always $40 \times 40 \mathrm{~cm}^{2}$ in the $\mathrm{x}$ and $\mathrm{z}$ dimensions whereas its depth was varied from 0 to $150 \mathrm{~cm}$ in the y dimension.

In the left panels of Figure 3 the Neutron Attenuation Factor is reported as a function of the weight of the TNT hidden in the trunk. The plot in the upper-left panel is obtained without specific selection of the tagging detector pixels. Consequently it correspond to a wide beam measurement. From the wide beam measurement, an rough image of the hidden object can be reconstructed by looking to the relative values of Attenuation Factor as a function of the tagging detector pixel.. By using this information, the Attenuation and the Asymmetry Factors are estimated by using only a subset of tagging detector pixels that are hitting the hidden sample in a narrow beam geometry. The results of the calculations in the narrow beam conditions are reported in the lower panels of Figure 3. It is seen that the inspection with narrow beam provides more precise information on the hidden explosive. Since an estimate of the lateral dimension of the hidden object can be reconstructed by the image, its weight can be obtained (assuming an average explosive material) by the Attenuation Factor up to about $100 \mathrm{~kg}$ and by the Asymmetry Factor for quantities larger than $200 \mathrm{~kg}$.

The discrimination between low $\mathrm{Z}$ compounds, as the explosives, and other materials is the main issue of the proposed system. As show in Figure 2, the Attenuation Factor alone is not sufficient to derive information about the presence of hidden threat materials, when the neutron interrogation is operated without additional information. In this case, the Asymmetry Factor provided the way of discriminate at least between low Z (explosives) and high Z (car engine) but, as shown in Figure 3, this works when the explosive mass is larger than $150 \mathrm{~kg}$. Only in this case, indeed, the value of the asymmetry is greater than the value obtained in other positions along the car. As an alternative, the neutronic interrogation can be used to implement other standard inspection techniques as the one based on x-rays. The fusion of the information between the two techniques is expected to solve ambiguities and lower the detectable explosive quantity. To some extent, a system using x-ray scan + neutron transmission for vehicle inspection is close to the one developed recently by CSIRO for airports described in ref.6.

Finally, some important considerations have to be done on the counting rates obtained with our set-up and on the dose equivalent absorbed by a driver passing through the scanning system. As already reported, each point of our simulation corresponds to the emission of $8 \times 10^{6}$ neutrons over $4 \pi$. If we suppose to have

- a $14 \mathrm{MeV}$ neutron generator emitting $8 \times 10^{7}$ neutron/s,

- neutron detectors with an efficiency of about $10 \%$ for $14 \mathrm{MeV}$ neutrons,

- a shielded neutron source,

with our geometry we will have a rate of $640 \times 10^{3}$ tagged neutron/s, $57 \times 10^{3}$ neutron/s detected by the $\mathrm{N}_{\mathrm{T}}$ detector in the case of an empty vehicle and $1.4 \times 10^{3}$ neutron/s detected by each segment of the $\mathrm{N}_{\mathrm{D}}$ detector. The count rate estimate for the scintillation detectors indicates that there is no need to use special electronics for the read-out of such system. 
Under the above conditions and considering a distance between the driver and the neutron producing target of about $50 \mathrm{~cm}$, the driver will receive a dose equivalent of about 0.8 microSv/s, when directly exposed to neutrons. Consequently, if the scanning time of the car is of the order of $10 \mathrm{~s}$, the driver will be exposed to the neutron flux for about $3 \mathrm{~s}$, which corresponds to a dose of about 2.5 microSv. This value is 400 times lower than the Italian annual allowed dose for not-exposed workers.

\section{Conclusions}

The results from Monte Carlo simulation reported here indicates that a fast scan of a vehicle can be performed by using transmitted and scattered neutrons. The sensitivity of this technique is limited to $150 \mathrm{~kg}$ to distinguish the type of material causing the absorption. The lowering of this threshold is certainly needed for a realistic use. In our opinion this goal can be simply achieved by using the neutronic system in combination with standard x-ray scan. The combination of the two techniques will lower the threshold thanks to the high resolution image obtained from the $\mathrm{x}$-rays. In those cases in where the X-ray penetration is poor, one can still have a useful information from the neutrons alone.

Moreover, problems related to the licensing of the neutronic system might be reduced when the neutron generator will not use radioactive tritium as in $\mathrm{D}+\mathrm{D}$ case. Monte Carlo calculations show indeed that an equivalent system is obtained by using $2.6 \mathrm{MeV}$ neutrons. In the latter case, however, the feasibility of a sealed neutron generator with an embedded system for associated particle detection has to be experimentally proved.

Finally it is worth mentioning that a conceptual design of an up-dated Neutronic Check point integrating the neutron system, the $\mathrm{x}$-ray scan with other technologies is now under discussion within the Preparatory Action for Security Research of the European Union.

\section{References}

[1] See as an example "Making the Nation Safer, the Role of Science and Technology in Countering Terrorism", edited by the Committee on Science and Technology for Countering Terrorism, The National Academy Press, Washington DC, USA, 2002 (www.nap.edu).

[2] See as an example http://europa.eu.int/comm/enterprise/security/index_en.htm.

[3] R. Speller, Radiation based security, Rad. Phys. And Chem. 61 (293) 2001 and references therein.

[4] T. Gozani, The role of neutron based inspection techniques in the post 9/11/01 era, Nucl. Instr. and Meth B 213 (460) 2004.

[5] G. Vourvopoulos and P. C. Womble, Pulsed fast/thermal neutron analysis: a technique for explosives detection, Talanta 54 (459) 2001.

[6] J.E. Eberhardt, S. Rainey, R.J. Stevens, B.D. Sowerby and J.R. Tickner, Fast neutron radiography scanner for the detection of contraband in air cargo containers, Applied Radiation and Isotopes 63 (179) 2005

[7] GEANT - Detector Description and Simulation Tool, CERN Program Library Long Write up W5013, 1994

[8] S. Pesente et al., Detection of hidden explosives by using tagged neutron beams with subnanosecond time resolution, Nucl. Instr. Meth. A 531 (657) 2004. 